PROCEEDINGS OF THE

AMERICAN MATHEMATICAL SOCIETY

Volume 138, Number 8, August 2010, Pages 2991-3001

S 0002-9939(10)10346-3

Article electronically published on April 8, 2010

\title{
RECONSTRUCTING A TOTALLY DISCONNECTED GROUPOID FROM ITS AMPLE SEMIGROUP
}

\author{
R. EXEL \\ (Communicated by Marius Junge)
}

\begin{abstract}
We show that a (not necessarily Hausdorff) étale, second countable groupoid $\mathcal{G}$ with totally disconnected unit space may be reconstructed solely from the algebraic structure of its ample semigroup $\mathcal{S}$. We also show that $C^{*}(\mathcal{G})$ possesses a universal property related to tight representations of $\mathcal{S}$.
\end{abstract}

\section{INTRODUCTION}

Recall that an open subset $S$ of a (not necessarily Hausdorff) étale groupoid $\mathcal{G}$ is said to be a slice (sometimes also a $\mathcal{G}$-set, or a bissection) if the domain and range maps are injective on $S$. The collection of all slices forms an inverse semigroup which has often been studied alongside the groupoid itself. Among other things the natural action of this semigroup on the unit space $\mathcal{G}^{(0)}$ highlights the dynamical nature of groupoids.

The set $\mathcal{S}$ formed by all compact (open) slices is called the ample semigroup of $\mathcal{G}$ [6. 2.10]. A compact slice $S$ is somewhat special because its characteristic function $1_{S}$ is in $C_{c}(G)$ and hence also in $C^{*}(\mathcal{G})$. We therefore get a map

$$
\rho: \mathcal{S} \rightarrow C^{*}(\mathcal{G}),
$$

defined by $\rho(S)=1_{S}$, which is multiplicative and satisfies $\rho\left(S^{-1}\right)=\rho(S)^{*}$. In other words, $\rho$ is a representation of $\mathcal{S}$ in $C^{*}(\mathcal{G})$.

From now on we will specialize to the situation in which $\mathcal{G}^{(0)}$ is totally disconnected. In this case one may prove that $\mathcal{G}$ contains many compact slices, enough to provide a basis for the topology of $\mathcal{G}$. The starting point for the present investigation was our interest in determining precisely how much information $\mathcal{S}$ contains about $\mathcal{G}$. In particular, one may ask the following:

1.2. Question. Is it possible to reconstruct $\mathcal{G}$ solely from the algebraic structure of the inverse semigroup $\mathcal{S}$ of compact slices?

The literature contains several constructions of groupoids associated to inverse semigroups, such as the associated groupoid described by Lawson in [4, Section 3.1], Kellendonk's topological groupoid [4, Section 9.2] or Paterson's groupoid of

Received by the editors September 17, 2009, and, in revised form, November 30, 2009.

2010 Mathematics Subject Classification. Primary 22A22, 20M18, 20M30, 46L55.

The author was partially supported by CNq. 
germs for the action described in [5. Proposition 4.3.2], but none of these give the expected result when the ample semigroup is fed as input.

The purpose of this paper is to give an affirmative answer to the above question, assuming $\mathcal{G}$ to be second countable, by using the theory of tight representations of semilattices and inverse semigroups introduced by the author in 1]. En passant we prove that the unitization of $C^{*}(\mathcal{G})$ is the universal $\mathrm{C}^{*}$-algebra for tight representations of $\mathcal{S}$ in the sense that these factor through the representation $\rho$ of (1.1).

Question (1.2) has already been addressed by Renault (see the paragraph immediately following [6, 2.10]), but under the assumption that the unit space of $\mathcal{G}$ is known. Without this crucial piece of information one is faced with the task of reconstructing the unit space of $\mathcal{G}$ from $\mathcal{S}$. Our strategy is to recover $\mathcal{G}^{(0)}$ from the idempotent semilattice $\mathcal{E}$ of $\mathcal{S}$ by means of its tight spectrum. The action of $\mathcal{S}$ on $\mathcal{G}^{(0)}$ is then easily recovered, and $\mathcal{G}$ pops out as the groupoid of germs for this action, as long as we use the appropriate notion of germs [5, page 140].

I would like to acknowledge an interesting conversation with Alex Kumjian in which the above question was raised and for which thanks are due.

\section{Preliminaries}

In this section we shall briefly survey the main results from [1 that we will need here. We will begin with some basic facts about inverse semigroups, all of which may be found in greater detail in many books on the subject such as [5] or 4]. References for groupoids may also be found in [6] in the Hausdorff case, in [3] in the general non-Hausdorff locally compact case, and in the first sections of [1], which is dedicated to the non-Hausdorff étale case, the situation which will concern us here.

Recall that a semigroup $\mathcal{S}$ is said to be an inverse semigroup if for every $s \in \mathcal{S}$ there exists a unique $s^{*} \in \mathcal{S}$ such that $s s^{*} s=s$ and $s^{*} s s^{*}=s^{*}$. An element $0 \in \mathcal{S}$ such that $0 s=s 0=0$, for all $s \in \mathcal{S}$, is said to be a zero element. All of our inverse semigroups will henceforth be assumed to contain a zero element.

An element $e \in \mathcal{S}$ is said to be idempotent if $e^{2}=e$. In this case $e$ is necessarily self-adjoint, meaning that $e^{*}=e$. The subset $\mathcal{E} \subseteq \mathcal{S}$ consisting of all idempotent elements is a commutative subsemigroup. It is also a semilattice under the order relation

$$
e \leq f \Longleftrightarrow e f=e, \quad \forall e, f \in \mathcal{E} .
$$

Given $e, f \in \mathcal{E}$, we say that $e$ is orthogonal to $f$, in symbols $e \perp f$, when $e f=0$. Otherwise we say that $e$ intersects $f$, writing $e \cap f$. Given subsets $Z, F \subseteq \mathcal{E}$, we will say that $Z$ is a cover [1, 11.5] for $F$, if $Z \subseteq F$ and for every nonzero $f \in F$ there exists $z \in Z$ such that $z$ ก $f$.

A representation of $\mathcal{S}$ in a unital $\mathrm{C}^{*}$-algebra $A$ is a map

$$
\pi: \mathcal{S} \rightarrow A,
$$

such that $\pi(s t)=\pi(s) \pi(t)$, and $\pi\left(s^{*}\right)=\pi(s)^{*}$, for all $s, t \in \mathcal{S}$, and such that $\pi(0)=0$. We shall moreover say that $\pi$ is tight $[1,11.6,13.1]$ if, whenever one is given finite subsets $X, Y \subseteq \mathcal{E}$ and a finite cover $Z$ for

$$
\mathcal{E}^{X, Y}:=\{f \in \mathcal{E}: f \leq x, \forall x \in X \text {, and } f \perp y, \forall y \in Y\},
$$


one has that

$$
\bigvee_{z \in Z} \pi(z)=\prod_{x \in X} \pi(x) \prod_{y \in Y}(1-\pi(y))
$$

Here " $\bigvee$ " refers to the operation of taking the supremum of a commuting set of projections (notice that the commutativity of $\mathcal{E}$ implies that the $\pi(z)$ commute with each other) which, in case of two projections $p$ and $q$, is defined by

$$
p \vee q=p+q-p q .
$$

The following is a central concept, implicit in [1]:

2.2. Definition. The tight $\mathrm{C}^{*}$-algebra of $\mathcal{S}$, denoted $C_{\text {tight }}^{*}(\mathcal{S})$, is the universal unital $\mathrm{C}^{*}$-algebra with one generator for each element of $\mathcal{S}$, subject to the relations stating that the standard map

$$
\pi_{u}: \mathcal{S} \rightarrow C_{\text {tight }}^{*}(\mathcal{S})
$$

is a tight representation. Henceforth $\pi_{u}$ will be referred to as the universal tight representation of $\mathcal{S}$.

The requirement that $C_{\text {tight }}^{*}(\mathcal{S})$ be unital is necessary because the right hand side of (2.1) explicitly mentions " 1 ". However, in case $\mathcal{E}$ admits no finite cover, (2.1) will never occur with $X \neq \emptyset$, and hence the right hand side of (2.1), once expanded out, will actually not involve "1". This phenomena has already been observed in [2, Section 8].

As is the case with universal objects, $\pi_{u}$ possesses the following universal property: given any tight representation $\pi$ of $\mathcal{S}$ in a unital $\mathrm{C}^{*}$-algebra $A$, there exists a unique unital *-homomorphism $\phi: C_{\text {tight }}^{*}(\mathcal{S}) \rightarrow A$, such that $\phi \circ \pi_{u}=\pi$.

In the remainder of this section we will briefly recall the description of $C_{\text {tight }}^{*}(\mathcal{S})$ as a groupoid $\mathrm{C}^{*}$-algebra given in 1 .

A representation of $\mathcal{E}$ in the algebra of complex numbers, say

$$
\phi: \mathcal{E} \rightarrow \mathbf{C},
$$

is called a character (without the assumption that $\phi(0)=0$; these are sometimes also called semicharacters). Since the elements of $\mathcal{E}$ are idempotent, the range of $\phi$ is contained in the set $\{0,1\}$, so $\phi$ is necessarily the characteristic function of some subset $\xi \subseteq \mathcal{E}$. One readily verifies that $0 \notin \xi$ and that $\xi$ is downwards directed as well as upwards hereditary. Such a $\xi$ is called a filter [1, 12.1], and it is easy to see that the characteristic function of any filter is a character. So there is a one-to-one correspondence between the set of all characters and the set of all filters, according to which we often identify one another.

An ultra-filter is a filter which is not properly contained in any filter, and we call the corresponding character an ultra-character. On the other hand, if a filter is associated to a tight character, we call it a tight filter.

With the topology inherited from the product topology on $\{0,1\}^{\mathcal{E}}$, the set $\widehat{\mathcal{E}}$ of nonzero characters is a locally compact space. The set of all tight characters is denoted $\widehat{\mathcal{E}}_{\text {tight }}$, and it happens to be precisely the closure [1,12.9] of the set of all ultra-characters within $\widehat{\mathcal{E}}$. We refer to $\widehat{\mathcal{E}}_{\text {tight }}$ as the tight spectrum of $\mathcal{E}$. 
If $s \in \mathcal{S}$ and if $\phi \in \widehat{\mathcal{E}}$ is such that $\phi\left(s^{*} s\right) \neq 0$, then the map

$$
\theta_{s}(\phi): e \in \mathcal{E} \mapsto \phi\left(s^{*} e s\right) \in\{0,1\}
$$

is a character satisfying $\left.\theta_{s}(\phi)\right|_{s s^{*}} \neq 0[1,10.3]$.

Denoting by $\mathcal{D}_{e}$ the set of all characters not vanishing on a given idempotent $e$, one may prove that $\theta_{s}$ defines a homeomorphism from $\mathcal{D}_{s^{*} s}$ to $\mathcal{D}_{s s^{*}}$. Collectively the family of partial homeomorphisms $\theta=\left\{\theta_{s}\right\}_{s \in \mathcal{S}}$ is an action [1, 4.3] of $\mathcal{S}$ on $\widehat{\mathcal{E}}$, which leaves $\widehat{\mathcal{E}}_{\text {tight }}$ invariant, in the sense that $\theta_{s}\left(\mathcal{D}_{s^{*} s} \cap \widehat{\mathcal{E}}_{\text {tight }}\right) \subseteq \widehat{\mathcal{E}}_{\text {tight }}$ [1, 12.11]. So we get, by restriction, an action of $\mathcal{S}$ on $\widehat{\mathcal{E}}_{\text {tight }}$.

For our purposes the relevant notion of germs [5, 1, 4.17] is defined as follows: if $s_{i} \in \mathcal{S}$ and if $\xi \in \mathcal{D}_{s_{i}^{*} s_{i}}$ for $i=1,2$, we say that $s_{1}$ and $s_{2}$ have the same germ 1 at $\xi$ if there exists $e \in \mathcal{E}$, such that $\xi \in \mathcal{D}_{e}$, and $s_{1} e=s_{2} e$. If $\xi \in \mathcal{D}_{s^{*} s}$, then the equivalence class for the relation "having the same germ" is called the germ of $s$ at $\xi$ and is denoted $[s, \xi]$. The groupoid of all germs for the action of $\mathcal{S}$ on $\widehat{\mathcal{E}}_{\text {tight }}$ is denoted $\mathcal{G}_{\text {tight }}=\mathcal{G}_{\text {tight }}(\mathcal{S})$. See [1] for more details.

The following is but a reinterpretation of [1, 13.3]. In it we will denote by $A^{\sim}$ the unitization of a $\mathrm{C}^{*}$-algebra $A$, with the convention that $A^{\sim}=A$, in case $A$ already has a unit.

2.4. Theorem. Given a countable inverse semigroup $\mathcal{S}$, there is a ${ }^{*}$-isomorphism

$$
\Lambda: C_{\text {tight }}^{*}(\mathcal{S}) \rightarrow C^{*}\left(\mathcal{G}_{\text {tight }}\right)^{\sim}
$$

such that, for every $s \in \mathcal{S}$,

$$
\Lambda\left(\pi_{u}(s)\right)=1_{\mathcal{X}_{s}},
$$

where the right hand side refers to the characteristic function of the compact slice

$$
\mathcal{X}_{s}=\left\{[s, \xi]: \xi \in \mathcal{D}_{s^{*} s}\right\},
$$

seen as an element of $C_{c}\left(\mathcal{G}_{\text {tight }}\right) \subseteq C^{*}\left(\mathcal{G}_{\text {tight }}\right)$. In addition, the closed ${ }^{*}$-subalgebra of $C^{*}\left(\mathcal{G}_{\text {tight }}\right)^{\sim}$ generated by the range of $\Lambda \circ \pi_{u}$ coincides with $C^{*}\left(\mathcal{G}_{\text {tight }}\right)$.

Proof. Initially notice that $\mathcal{X}_{s}$ is a slice of $\mathcal{G}_{\text {tight }}$ by [4, 41.8], which is compact by [1. 4.15], since $\mathcal{D}_{s^{*} s}$ is compact, as argued in the second paragraph after [1, 10.2]. Therefore the characteristic function of $\mathcal{X}_{s}$ is in $C_{c}\left(\mathcal{G}_{\text {tight }}\right) \subseteq C^{*}\left(\mathcal{G}_{\text {tight }}\right)$.

We next claim that the set $\left\{1_{\mathcal{X}_{s}}: s \in \mathcal{S}\right\}$ generates $C^{*}\left(\mathcal{G}_{\text {tight }}\right)$ as a $\mathrm{C}^{*}$-algebra. Denoting by $A$ the closed ${ }^{*}$-subalgebra generated by this set, notice that, in view of [1, 3.10], the claim will follow once we prove that $C_{c}\left(\mathcal{X}_{s}\right) \subseteq A$ for all $s \in \mathcal{S}$.

If $f \in C_{c}\left(\mathcal{X}_{s}\right)$, let $g$ be the unique element of $C_{c}\left(\mathcal{X}_{s^{*} s}\right)$ such that $g(d(\gamma))=f(\gamma)$, for all $\gamma \in \mathcal{X}_{s}$, where $d$ refers to the source map. Since $f$ coincides with the product of $1_{\mathcal{X}_{s}}$ and $g$ in $C^{*}\left(\mathcal{G}_{\text {tight }}\right)$, our claim will be proved once we show that $g \in A$.

Using [1, 4.16] and the Stone-Weierstrass Theorem we have that $C_{0}\left(\mathcal{G}_{\text {tight }}^{(0)}\right)$ is generated by the set $\left\{1_{\mathcal{X}_{e}}: e \in \mathcal{E}\right\}$, so

$$
g \in C_{c}\left(\mathcal{X}_{s^{*} s}\right) \subseteq C_{0}\left(\mathcal{G}_{\text {tight }}^{(0)}\right) \subseteq A .
$$

This proves our claim.

Viewing $C_{\text {tight }}^{*}(\mathcal{S})$ as an algebra of operators on a Hilbert space $H$ via some faithful representation, $\pi_{u}$ may be regarded as a tight representation of $\mathcal{S}$ on $H$.

\footnotetext{
${ }^{1}$ Except for the case of topologically free actions, this notion of germs is not the same as the one which emphasizes an open set containing $\xi$ where $\theta_{s_{1}}$ and $\theta_{s_{2}}$ agree.
} 
Applying [1, 13.3], we deduce that there is a *-representation $\psi$ of $C^{*}\left(\mathcal{G}_{\text {tight }}\right)$ on $H$ such that

$$
\psi\left(1_{\mathcal{X}_{s}}\right)=\pi_{u}(s), \quad \forall s \in \mathcal{S}
$$

(notice that the argument of $\rho$ in $[1,10.15]$ is precisely $1_{\mathcal{X}_{s}}$ in the notation adopted here). By the claim we deduce that the range of $\psi$ is contained in $C_{\text {tight }}^{*}(\mathcal{S})$, so we may view $\psi$ as a ${ }^{*}$-homomorphism

$$
\psi: C^{*}\left(\mathcal{G}_{\text {tight }}\right) \rightarrow C_{\text {tight }}^{*}(\mathcal{S}) .
$$

We next want to extend this to unitizations, but, while $C_{\text {tight }}^{*}(\mathcal{S})$ is unital by definition, $C^{*}\left(\mathcal{G}_{\text {tight }}\right)$ may or may not be unital. However, we claim that if $C^{*}\left(\mathcal{G}_{\text {tight }}\right)$ is unital, then

$$
\psi(1)=1 .
$$

Assuming that $C^{*}\left(\mathcal{G}_{\text {tight }}\right)$ is unital, it follows that $\mathcal{G}_{\text {tight }}^{(0)}$ is compact and hence, by [1. 4.16], $\widehat{\mathcal{E}}_{\text {tight }}$ is also compact. Since $\left\{\mathcal{D}_{e}\right\}_{e \in \mathcal{E}}$ is a cover for $\widehat{\mathcal{E}}_{\text {tight }}$, we may extract a finite subcover, say

$$
\widehat{\mathcal{E}}_{\text {tight }}=\mathcal{D}_{e_{1}} \cup \ldots \cup \mathcal{D}_{e_{n}} \text {. }
$$

The unit of $C^{*}\left(\mathcal{G}_{\text {tight }}\right)$ is then given by

$$
1=\bigvee_{k=1}^{n} 1_{\mathcal{X}_{e_{k}}}
$$

Given any nonzero $e \in \mathcal{E}$, choose an ultra-filter $\xi$ containing $e$, as done in 1, Section 12], and observe that $\xi \in \widehat{\mathcal{E}}_{\text {tight }}$, by [1, 12.7]. Therefore $\xi \in \mathcal{D}_{e_{k}}$ for some $k$, and hence $e_{k} \in \xi$ so that $e$ ก $e_{k}$. In other words, $\left\{e_{1}, \ldots, e_{n}\right\}$ is a cover for $\mathcal{E}$ in the sense of [1, 11.5].

Writing $\mathcal{E}=\mathcal{E}^{X, Y}$, where $X=\emptyset$ and $Y=\{0\}$, and observing that $\pi_{u}$ is tight by definition, we deduce that

$$
\bigvee_{k=1}^{n} \pi_{u}\left(e_{k}\right)=\prod_{x \in X} \pi_{u}(x) \prod_{y \in Y}\left(1-\pi_{u}(y)\right)=1 .
$$

So,

$$
\psi(1) \stackrel{\sqrt[2.4 .3]{=}}{=} \bigvee_{k=1}^{n} \psi\left(1_{\mathcal{X}_{e_{k}}}\right) \stackrel{(2.4 .2)}{=} \bigvee_{k=1}^{n} \pi_{u}\left(e_{k}\right)=1
$$

Being a unital algebra, $C^{*}\left(\mathcal{G}_{\text {tight }}\right)$ coincides with its unitization and $\psi$ may be viewed as a unital *-homomorphism

$$
\widetilde{\psi}: C^{*}\left(\mathcal{G}_{\text {tight }}\right)^{\sim} \rightarrow C_{\text {tight }}^{*}(\mathcal{S}) .
$$

On the other hand, should $C^{*}\left(\mathcal{G}_{\text {tight }}\right)$ lack a unit, the natural extension of $\psi$ to its unitization again provides a unital homomorphism $\widetilde{\psi}$ as above. Thus $\widetilde{\psi}$ is available whatever the case may be.

Changing tack, consider the map

$$
\rho: s \in \mathcal{S} \mapsto 1_{\mathcal{X}_{s}} \in C^{*}\left(\mathcal{G}_{\text {tight }}\right)^{\sim} .
$$

Either using [1, 13.3] or by direct computation one proves that $\rho$ is a tight representation of $\mathcal{S}$, in which case the universal property of $C_{\text {tight }}^{*}(\mathcal{S})$ intervenes, providing a unital *-homomorphism

$$
\Lambda: C_{\text {tight }}^{*}(\mathcal{S}) \rightarrow C^{*}\left(\mathcal{G}_{\text {tight }}\right)^{\sim}
$$


such that

$$
\Lambda\left(\pi_{u}(s)\right)=\rho(s)=1_{\mathcal{X}_{s}}, \quad \forall s \in \mathcal{S} .
$$

Noticing that $C_{\text {tight }}^{*}(\mathcal{S})$ is generated by $\left\{\pi_{u}(s): s \in \mathcal{S}\right\} \cup\{1\}$, that $C^{*}\left(\mathcal{G}_{\text {tight }}\right)^{\sim}$ is generated by $\left\{1_{\mathcal{X}_{s}}: s \in \mathcal{S}\right\} \cup\{1\}$, and contrasting (2.4.2) with (2.4.4), we deduce that $\Lambda$ and $\widetilde{\psi}$ are each other's inverse. In particular, $\Lambda$ is a ${ }^{*}$-isomorphism.

The last sentence of the statement now follows immediately from the claim at the beginning of the proof.

\section{Totally Disconnected SPACES}

Throughout this section we will fix a Hausdorff topological space $X$ and a basis $\mathcal{B}$ for the topology of $X$ consisting of compact open sets. Evidently, in order for such a basis to exist, $X$ must be locally compact and totally disconnected.

Replacing $\mathcal{B}$ by the collection of all finite intersections of members of $\mathcal{B}$, we may assume that $\mathcal{B}$ is closed under intersections. We will also suppose that the empty set is a member of $\mathcal{B}$ (which is necessarily the case if $X$ has more than one point). Summarizing, $\mathcal{B}$ is a basis for the topology of $X$ such that

- if $U \in \mathcal{B}$, then $U$ is compact and open;

- $\emptyset \in \mathcal{B}$;

- if $U, V \in \mathcal{B}$, then $U \cap V \in \mathcal{B}$.

With the usual order of inclusion it is clear that $\mathcal{B}$ is a semilattice with zero, the role of the latter being played by the empty set. This semilattice structure of $\mathcal{B}$ will be our main concern from now on, and it is our purpose to show that its tight spectrum is homeomorphic to $X$.

If $\mathcal{F}$ is any collection of subsets of $X$ we will adopt the notation $\bigcap \mathcal{F}$ to refer to the intersection of all members of $\mathcal{F}$, namely

$$
\bigcap^{\mathcal{F}}=\bigcap_{A \in \mathcal{F}} A \text {. }
$$

This is, in fact, the standard convention for the intersection operator in set theory.

3.2. Lemma. Let $X$ be a topological space, let $\mathcal{F}$ be a family of closed subsets of $X$, and let $U$ be an open subset of $X$ such that

$$
\bigcap \mathcal{F} \subseteq U
$$

Suppose moreover that at least one member of $\mathcal{F}$ is compact. Then there are finitely many $F_{1}, F_{2}, \ldots, F_{n} \in \mathcal{F}$ such that $F_{1} \cap F_{2} \cap \ldots \cap F_{n} \subseteq U$.

Proof. Let $G \in \mathcal{F}$ be compact and consider the following collection of compact subsets of $X$ :

$$
\mathcal{F}^{\prime}=\{F \cap G \cap(X \backslash U): F \in \mathcal{F}\}
$$

It is clear that

$$
\bigcap \mathcal{F}^{\prime} \subseteq \bigcap \mathcal{F} \subseteq U
$$

but since $\bigcap \mathcal{F}^{\prime}$ is also contained in $X \backslash U$, we see that $\bigcap \mathcal{F}^{\prime}=\emptyset$. By compactness there are finitely many $F_{1}, F_{2}, \ldots, F_{n} \in \mathcal{F}$ such that $F_{1} \cap F_{2} \cap \ldots \cap F_{n} \cap G \cap(X \backslash U)=$ $\emptyset$, and hence

$$
F_{1} \cap F_{2} \cap \ldots \cap F_{n} \cap G \subseteq U
$$


Given a point $x$ in $X$, the set

$$
\xi_{x}=\{U \in \mathcal{B}: U \ni x\}
$$

is evidently an ultra-filter and hence also a tight filter [1, 12.7]. The converse of this statement is in order.

3.4. Proposition. If $\xi$ is a tight filter on $\mathcal{B}$, then there exists a unique $x$ in $X$ such that $\xi=\xi_{x}$. In other words, the correspondence

$$
\Phi: x \in X \mapsto \xi_{x} \in \widehat{\mathcal{B}}_{\text {tight }}
$$

is a bijection.

Proof. Because $\xi$ is a filter it has the finite intersection property, and, since the members of $\xi$ are compact sets, we see that $\bigcap \xi$ (notation as in (3.1)) is nonempty.

Our first claim is that $\bigcap \xi$ consists of a single point. To prove this assume otherwise, and for each $x \in \bigcap \xi$ choose an open set $W_{x}$ such that

$$
x \in W_{x} \nsupseteq \bigcap \xi
$$

(to obtain such a set one may use the Hausdorff property to separate $x$ from any other point of $\bigcap \xi$ ). Since $\mathcal{B}$ is a basis for the topology of $X$, we might as well take $W_{x} \in \mathcal{B}$. The $W_{x}$ therefore form a cover for $\bigcap \xi$, and, since the latter is compact, we may choose a finite subcover, say

$$
\bigcap \xi \subseteq W_{x_{1}} \cap \ldots \cap W_{x_{k}}
$$

Employing Lemma 3.2 we deduce that there are finitely many $F_{1}, \ldots, F_{n} \in \xi$ such that

$$
V:=F_{1} \cap \ldots \cap F_{n} \subseteq W_{x_{1}} \cap \ldots \cap W_{x_{k}} .
$$

Since $\xi$ is a filter, and hence closed under finite intersections, we see that $V \in \xi$. It is therefore evident that

$$
\left\{W_{x_{1}} \cap V, \ldots, W_{x_{k}} \cap V\right\}
$$

is a cover for $V$ in the technical sense of [1, 11.5]. If $\phi$ is the character of $\mathcal{B}$ associated to $\xi$, as in [1, 12.6], we therefore have by (2.1) that

$$
\bigvee_{i=1}^{k} \phi\left(W_{x_{1}} \cap V\right)=\phi(V)
$$

Given that $V \in \xi$, the right hand side above equals 1 , and hence there exists some $i$ such that $W_{x_{i}} \cap V \in \xi$, so that also $W_{x_{i}} \in \xi$. It follows that $W_{x_{i}} \supseteq \bigcap \xi$, contradicting (3.4.1) and hence proving our claim.

We conclude that $\bigcap \xi=\left\{x_{0}\right\}$ for some $x_{0}$ in $X$, and the proof will be finished once we prove that $\xi=\xi_{x_{0}}$. Since the inclusion $\xi \subseteq \xi_{x_{0}}$ is trivial, we concentrate on the opposite one. Given $U \in \xi_{x_{0}}$, notice that

$$
\bigcap \xi=\left\{x_{0}\right\} \subseteq U
$$

so we may invoke Lemma 3.2 once more to produce $F_{1}, \ldots, F_{n} \in \xi$, such that

$$
W:=F_{1} \cap \ldots \cap F_{n} \subseteq U .
$$

Again we have that $W \in \xi$ so that also $U \in \xi$, concluding the proof that $\xi_{x_{0}} \subseteq \xi$.

The fact that $x_{0}$ is uniquely determined follows from the Hausdorff property of $X$. 
3.5. Proposition. If $U \in \mathcal{B}$, then the image of $U$ under the map $\Phi$ of (3.4) coincides with the set

$$
\mathcal{D}_{U}:=\left\{\xi \in \widehat{\mathcal{B}}_{\text {tight }}: \xi \ni U\right\} .
$$

Proof. For $x \in X$ we have that

$$
x \in U \Longleftrightarrow \xi_{x} \ni U \Longleftrightarrow \xi_{x} \in \mathcal{D}_{U} \Longleftrightarrow \Phi(x) \in \mathcal{D}_{U} .
$$

We may now prove the main result of this section:

3.6. Theorem. Let $X$ be a Hausdorff topological space and let $\mathcal{B}$ be a basis for the topology of $X$ consisting of compact open subsets, and which is moreover closed under finite intersections. Assuming that $\emptyset \in \mathcal{B}$ and viewing $\mathcal{B}$ as a semilattice, the correspondence

$$
\Phi: x \in X \mapsto \xi_{x} \in \widehat{\mathcal{B}}_{\text {tight }}
$$

gives a homeomorphism from $X$ to the tight spectrum of $\mathcal{B}$.

Proof. After (3.4) all that remains to be proved is that $\Phi$ is continuous and open. A basic open set in $\widehat{\mathcal{B}}_{\text {tight }}$ is of the form

$$
\Omega=\left\{\xi \in \widehat{\mathcal{B}}_{\text {tight }}: U_{1}, \ldots, U_{n} \in \xi ; V_{1}, \ldots, V_{m} \notin \xi\right\},
$$

where $U_{1}, \ldots, U_{n} ; V_{1}, \ldots, V_{m} \in \mathcal{B}$. With notation as in (3.5) notice that

$$
\Omega=\bigcap_{i=1}^{n} \mathcal{D}_{U_{i}} \cap \bigcap_{j=1}^{m} \widehat{\mathcal{B}}_{\text {tight }} \backslash \mathcal{D}_{V_{i}}
$$

so by (3.5) we have

$$
\Phi^{-1}(\Omega)=\bigcap_{i=1}^{n} U_{i} \cap \bigcap_{j=1}^{m} X \backslash V_{i},
$$

which is an open subset of $X$, proving the continuity of $\Phi$. To prove that $\Phi$ is an open map it suffices to verify that $\Phi(U)$ is open in $\widehat{\mathcal{B}}_{\text {tight }}$, for all $U \in \mathcal{B}$, but this follows at once from (3.5).

\section{Groupoids With totally Disconnected unit SPACE}

In this section we will fix a (not necessarily Hausdorff) étale groupoid $\mathcal{G}$, with range map denoted $r$ and domain (or source) map written $d$. For a precise definition see, e.g., [5] or [1, 3.1].

Recall that a slice of $\mathcal{G}$ is any open subset $S \subseteq \mathcal{G}$, such that $r$ and $d$ are injective when restricted to $S$. It is well known, and easy to prove, that the collection of all slices forms a basis for the topology of $\mathcal{G}$.

If $S$ and $T$ are slices, their product

$$
S T=\{\sigma \tau: \sigma \in S, \tau \in T, d(\sigma)=r(\tau)\}
$$

may be shown to be a slice. With this multiplication operation the collection of all slices forms a semigroup, and in fact an inverse semigroup, where the inverse of a slice $S$ is given by

$$
S^{*}=\left\{\sigma^{-1}: \sigma \in S\right\} .
$$

By trivial reasons every open subset of $\mathcal{G}^{(0)}$ (the unit space of $\mathcal{G}$ ) is a slice, so the inverse semigroup of all slices contains the topology of $\mathcal{G}^{(0)}$. The latter is in fact identical to the idempotent semilattice of the former. 
Recall that a topological space is said to be totally disconnected when its topology admits a basis formed by subsets which are both open and closed.

4.1. Proposition. If $\mathcal{G}^{(0)}$ is totally disconnected, then the collection of all compact slices forms a basis for the topology of $\mathcal{G}$.

Proof. Given $\gamma \in U \subseteq \mathcal{G}$, with $U$ open, we need to find a compact slice $T$ such that

$$
\gamma \in T \subseteq U \text {. }
$$

Choose a slice $S$ such that $\gamma \in S \subseteq U$, and notice that since $d(\gamma) \in d(S)$ and since $d(S)$ is open, there exists a compact2 open subset $K$ of $\mathcal{G}^{(0)}$ such that $d(\gamma) \in$ $K \subseteq d(S)$. Observing that $d$ is a homeomorphism from $S$ to $d(S)$, we see that $T:=S \cap d^{-1}(K)$ is a compact slice satisfying (4.1.1).

Since we are not assuming $\mathcal{G}$ to be Hausdorff, the compact slices provided by the above result might not be closed subsets. We have therefore fallen short of proving that $\mathcal{G}$ is totally disconnected, at least according to the classical definition of this concept which requires a basis consisting of subsets which are both open and closed. I wonder if the definition of totally disconnected non-Hausdorff spaces should be adapted to include spaces such as our $\mathcal{G}$ above.

It is easy to prove that the product of two compact slices is also compact, so the collection of all compact slices forms a semigroup. It is clearly also an inverse semigroup since $S^{*}$ is easily seen to be compact should $S$ be compact.

In order to proceed we need the following elementary result in General Topology, which is perhaps well known to the reader:

4.2. Lemma. A (not necessarily Hausdorff) second countable topological space contains at most countably many compact open subsets.

Proof. Let $\mathcal{B}$ be a countable basis for the topology of our space. Given a compact open subset $K$, for each $x \in K$, pick $U_{x} \in \mathcal{B}$ such that $x \in U_{x} \subseteq K$. Thus $\left\{U_{x}\right\}_{x \in K}$ is an open cover for $K$, from which we may extract a finite subcover, say $\left\{U_{x_{i}}\right\}_{i=1}^{n}$. Consequently $K$ is the union of finitely many members of $\mathcal{B}$. Since there are only countably many finite subsets of $\mathcal{B}$, the result follows.

If we assume that, besides satisfying the hypothesis of (4.1), our groupoid is second countable, it will follow that the inverse semigroup of all compact slices is countable.

Summarizing, we have:

4.3. Corollary. Let $\mathcal{G}$ be a (not necessarily Hausdorff) étale, second countable groupoid with totally disconnected unit space. Then there is a (necessarily) countable inverse semigroup $\mathcal{S}$ consisting of compact slices of $\mathcal{G}$ which is moreover a basis for the topology of $\mathcal{G}$.

Proof. The collection $\mathcal{S}$ formed by all compact slices fits the above description.

From now on we fix a countable inverse semigroup $\mathcal{S}$ consisting of compact slices of $\mathcal{G}$ which is a basis for the topology of $\mathcal{G}$, and let us denote by $\mathcal{E}$ the idempotent semilattice of $\mathcal{S}$. Each member of $\mathcal{E}$ is therefore a compact open subset of $\mathcal{G}^{(0)}$.

\footnotetext{
${ }^{2}$ Notice that the axioms for étale groupoids include the requirements that $\mathcal{G}^{(0)}$ be Hausdorff and locally compact. With the added assumption that $\mathcal{G}^{(0)}$ is totally disconnected, one may easily prove that the collection of all open compact subsets is a basis for the topology of $\mathcal{G}^{(0)}$.
} 
It is also clear that $\mathcal{E}$ is a basis for the topology of $\mathcal{G}^{(0)}$, so by (3.6) we obtain a homeomorphism

$$
\Phi: \mathcal{G}^{(0)} \rightarrow \widehat{\mathcal{E}}_{\text {tight }}
$$

such that $\Phi(U)=\mathcal{D}_{U}$, for all $U \in \mathcal{E}$, according to (3.5).

Recall from [1, 5.3] that $\mathcal{S}$ acts on $\mathcal{G}^{(0)}$ as follows: given $S \in \mathcal{S}$,

$$
\lambda_{S}: d(S) \rightarrow r(S)
$$

is defined to be the homeomorphism given, for every $x \in d(S)$, by

$$
\lambda_{S}(x)=r(\gamma),
$$

where $\gamma$ is the unique element in $S$ such that $d(\gamma)=x$. This action was denoted by $\theta$ in [1], but we denote it here by $\lambda$ in order to distinguish it from the action of $\mathcal{S}$ on its tight spectrum, as in (2.3).

4.5. Lemma. Let $S$ and $U$ be slices of $\mathcal{G}$ with $U \subseteq \mathcal{G}^{(0)}$. Then, for every $\gamma \in S$, one has that $d(\gamma) \in S^{*} U S$ if and only if $r(\gamma) \in U$.

Proof. Assuming that $d(\gamma) \in S^{*} U S$, write $d(\gamma)=\alpha^{-1} y \beta$, with $\alpha, \beta \in S$ and $y \in U$. Then $d(\gamma)=d(\beta)$, and hence $\gamma=\beta$, because $d$ is injective on $S$. It follows that

$$
r(\gamma)=r(\beta)=d(y)=y \in U .
$$

Conversely, if $r(\gamma) \in U$, then

$$
d(\gamma)=\gamma^{-1} \gamma=\gamma^{-1} r(\gamma) \gamma \in S^{*} U S .
$$

4.6. Proposition. The homeomorphism $\Phi$ in (4.4) establishes an equivalence between $\lambda$ and $\theta$. More precisely, for every $S \in \mathcal{S}$ the diagram below commutes:

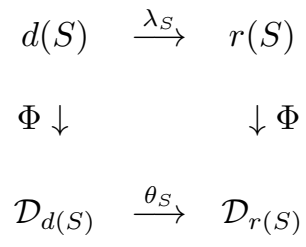

Proof. Let $x \in d(S)$, and write $x=d(\gamma)$, with $\gamma \in S$. Then, using brackets to denote boolean value, for every $U \in \mathcal{E}$ one has

$$
\begin{aligned}
\left.\theta_{S}(\Phi(x))\right|_{U}= & \left.\Phi(x)\right|_{S^{*} U S}=\left[d(\gamma) \in S^{*} U S\right] \stackrel{(4.5)}{=}[r(\gamma) \in U] \\
& =\left.\Phi(r(\gamma))\right|_{U}=\left.\Phi\left(\lambda_{S}(x)\right)\right|_{U} .
\end{aligned}
$$

It is evident that the equivalent actions of $\mathcal{S}$ above give rise to isomorphic groupoid of germs, so the groupoid of germs for the action $\lambda$, which we will temporarily denote by $\mathcal{H}$, is isomorphic to the groupoid of germs for the action $\theta$, which we have denoted by $\mathcal{G}_{\text {tight }}$ in section 2 The obvious isomorphism, say $f: \mathcal{H} \rightarrow \mathcal{G}_{\text {tight }}$, is then given by $f([S, x])=[S, \Phi(x)]$, for all $S \in \mathcal{S}$ and $x \in d(S)$.

In addition, recall from [1, 5.4] that $\mathcal{H}$ is isomorphic to $\mathcal{G}$, under an isomorphism $g: \mathcal{H} \rightarrow \mathcal{G}$ which sends the germ $[S, x]$ to the unique element $\gamma \in S$ such that $d(\gamma)=x$. We thus get an isomorphism from $\mathcal{G}_{\text {tight }}$ to $\mathcal{G}$ by composition, namely

$$
\mathcal{G}_{\text {tight }} \stackrel{f^{-1}}{\rightarrow} \mathcal{H} \stackrel{g}{\rightarrow} \mathcal{G} .
$$

This shows that $\mathcal{G}$ may be recovered from $\mathcal{S}$, a result which deserves to be highlighted in the following: 
4.8. Theorem. Let $\mathcal{G}$ be a (not necessarily Hausdorff) étale, second countable groupoid with totally disconnected unit space. Also let $\mathcal{S}$ be a (necessarily) countable inverse semigroup consisting of compact slices of $\mathcal{G}$ which is a basis for the topology of $\mathcal{G}$. Then $\mathcal{G}$ is isomorphic to the groupoid $\mathcal{G}_{\text {tight }}$ formed by the germs of the canonical action of $\mathcal{S}$ on the tight spectrum of its idempotent semilattice.

Given $S \in \mathcal{S}$, let $\mathcal{X}_{S} \subseteq \mathcal{G}_{\text {tight }}$ be given by

$$
\mathcal{X}_{S}=\left\{[S, \xi]: \xi \in \mathcal{D}_{d(S)}\right\},
$$

and recall that the characteristic function $1_{\mathcal{X}_{S}}$ was referred to in (2.4) as being equal to $\Lambda\left(\pi_{u}(S)\right)$. We are interested in determining the image of $\mathcal{X}_{S}$ under $g \circ f^{-1}$. Clearly $f^{-1}\left(\mathcal{X}_{S}\right)=\{[S, x]: x \in d(S)\}$, whence $g\left(f^{-1}\left(\mathcal{X}_{S}\right)\right)=S$. Thus, if we let

$$
\Gamma: C^{*}\left(\mathcal{G}_{\text {tight }}\right) \rightarrow C^{*}(\mathcal{G})
$$

be the ${ }^{*}$-isomorphism induced from (4.7), we conclude from (2.4) that

$$
\Gamma\left(\Lambda\left(\pi_{u}(S)\right)\right)=\Gamma\left(1_{\mathcal{X}_{s}}\right)=1_{S} .
$$

We have therefore proven the following.

4.9. Theorem. Let $\mathcal{G}$ be a (not necessarily Hausdorff) étale, second countable groupoid with totally disconnected unit space. Also let $\mathcal{S}$ be a (necessarily) countable inverse semigroup consisting of compact slices of $\mathcal{G}$ which is a basis for the topology of $\mathcal{G}$. Then there is a ${ }^{*}$-isomorphism

$$
\psi: C_{\text {tight }}^{*}(S) \rightarrow C^{*}(\mathcal{G})^{\sim}
$$

such that $\psi \circ \pi_{u}(S)=1_{S}$, for all $S \in \mathcal{S}$.

Proof. It is enough to take $\psi=\widetilde{\Gamma} \circ \Lambda$, where $\widetilde{\Gamma}$ is the extension of $\Gamma$ to the corresponding unitized algebras.

Borrowing from the universal property of $C_{\text {tight }}^{*}(S)$ we have the following main consequence:

4.10. Corollary. Let $\mathcal{G}$ and $\mathcal{S}$ be as above. Then for every tight representation $\pi$ of $\mathcal{S}$ in a unital $C^{*}$-algebra $A$, there exists a unique unital ${ }^{*}$-homeomorphism $\phi: C^{*}(\mathcal{G})^{\sim} \rightarrow A$ such that $\phi\left(1_{S}\right)=\pi(S)$, for every $S \in \mathcal{S}$.

\section{REFERENCES}

[1] R. Exel, Inverse semigroups and combinatorial C*-algebras, Bull. Braz. Math. Soc. (N.S.) 39 (2008), 191-313. [arXiv:math.OA/0703182] MR2419901 (2009b:46115)

[2] R. Exel and M. Laca, Cuntz-Krieger algebras for infinite matrices, J. reine angew. Math. 512 (1999), 119-172. [arXiv:funct-an/9712008] MR1703078 (2000i:46064)

[3] M. Khoshkam and G. Skandalis, Regular representation of groupoid $C^{*}$-algebras and applications to inverse semigroups, J. Reine Angew. Math. 546 (2002), 47-72. MR1900993 (2003f:46084)

[4] M. V. Lawson, Inverse semigroups, the theory of partial symmetries, World Scientific, 1998. MR.1694900 (2000g:20123)

[5] A. L. T. Paterson, Groupoids, inverse semigroups, and their operator algebras, Birkhäuser, 1999. MR1724106 (2001a:22003)

[6] J. Renault, A groupoid approach to $C^{*}$-algebras, Lecture Notes in Mathematics, vol. 793, Springer, 1980. MR.584266 (82h:46075)

Departamento de Matemática, Universidade Federal de Santa Catarina, 88040-900, Florianópolis, Brasil

E-mail address: r@exel.com.br 